\title{
Spin-Hall transport of heavy holes in III-V semiconductor quantum wells
}

\author{
John Schliemann and Daniel Loss \\ Department of Physics and Astronomy, University of Basel, CH-4056 Basel, Switzerland \\ (Received 19 May 2004; revised manuscript received 20 September 2004; published 9 February 2005)
}

\begin{abstract}
We investigate spin transport of heavy holes in III-V semiconductor quantum wells in the presence of spin-orbit coupling of the Rashba type due to structure-inversion asymmetry. Similarly to the case of electrons, the longitudinal spin conductivity vanishes, whereas the off-diagonal elements of the spin-conductivity tensor are finite giving rise to an intrinsic spin-Hall effect. For a clean system we find a closed expression for the spin-Hall conductivity depending on the length scale of the Rashba coupling and the hole density. In this limit the spin-Hall conductivity is enhanced compared to its value for electron systems, and it vanishes with increasing strength of the impurity scattering. As an aside, we also derive explicit expressions for the Fermi momenta and the densities of holes in the different dispersion branches as a function of the spin-orbit coupling parameter and the total hole density. These results are of relevance for the interpretation of possible Shubnikov-de Haas measurements detecting the Rashba spin splitting.
\end{abstract}

DOI: 10.1103/PhysRevB.71.085308

PACS number(s): 73.63. $-\mathrm{b}, 85.75 .-\mathrm{d}$

\section{INTRODUCTION}

In the last years, the emerging field of spin electronics has become a major branch of solid state physics and comprises by now all kinds of spin-dependent phenomena in semiconductor structures and related systems. ${ }^{1-3}$ Most recently, the possibility of spin-Hall currents has attracted considerable theoretical interest. ${ }^{4-19}$ In these studies a spin current (as opposed to a charge current) driven by an electric field perpendicular to it was investigated, where the spinful intinerant charge carrriers are bulk valence-band holes in III-V zinc blende semiconductors ${ }^{4-9}$ or conduction band electrons in quantum wells ${ }^{10-19}$ of the same type of materials. In the present work we extend these studies to the case of heavy holes in quantum wells being subect to spin-orbit coupling of the Rashba type due to structure-inversion asymmetry. ${ }^{20-24}$

From a historical perspective, the notion of the spin-Hall effect in systems of itinerant spinful charge carriers was considered first by Dyakonov and Perel ${ }^{25}$ in the early 1970's, and in a more recent paper by Hirsch. ${ }^{26}$ In these studies the predicted spin-Hall effect is due to spin-orbit effects influencing scattering processes upon static impurities. Following the terminology used in Refs. 10 and 5 this is referred to as the extrinsic spin-Hall effect since it necessarily requires spin-dependent impurity scattering. This is in contrast to the intrinsic spin-Hall effect studied theoretically very recently in Refs. 4-19. which is entirely due to spin-orbit coupling terms in the single-particle carrier Hamiltonian and occurs even in the absence of any scattering process. We note that this distinction between intrinsic and extrinsic effects becomes ambiguous in the limit of weak spin-orbit coupling when life time effects of carrier quasiparticles have to be taken into account. ${ }^{11}$

Yet another type of spin-Hall effect was studied recently by Meier and $\operatorname{Loss}^{27}$ in a two-dimensional Heisenberg model consisting of nonitinerant spins, in contrast to the itinerantcarrier systems mentioned before. In the case of conductionband electrons in III-V semiconductor quantum wells, the intrinsic spin-Hall effect results from spin-orbit coupling of the Rashba type (due to structure-inversion asymmetry ${ }^{28}$ ) and/or the Dresselhaus type (due to bulk-inversion asymmetry $\left.{ }^{29}\right)$. Moreover, the interplay of these two effects leads to particularly interesting transport effects. ${ }^{30,31,12}$ For bulk valence band holes the effects of spin orbit coupling underlying the intrinsic spin-Hall effect are incorporated in Luttinger's effective Hamiltonian ${ }^{32}$ giving rise to two different dispersion branches differing in their effective masses and known as heavy and light holes. Within this description, these states form for a given wave vector multiplets of total angular momentum $3 / 2$ resulting from the $s=1 / 2$ spin degree of freedom of the holes and the $l=1$ orbital anglar momentum of the $p$-type atomic orbitals forming the valence band. ${ }^{33}$ In a quantum well, a splitting between the heavy and light holes occurs due to the size quantization in the growth direction of the heterostructure. For a sufficiently narrow quantum well and for not too high densities and temperatures, only the lowest heavy hole subbands are significantly occupied. Here the angular momentum of the heavy hole states points predominantly along the growth direction, ${ }^{21}$ corresponding to the total angular momentum states $\pm 3 / 2$, an approximation we shall adopt in the present work.

Experimental investigations of such systems include studies of spin polarization and transitions to an insulating state induced by magnetic fields. ${ }^{34}$ Moreover, the spin splitting due to spin-orbit coupling has been studied in detail via Shubnikov-de Haas oscillations ${ }^{35,36}$ including also anisotropic properties of the magnetoresistance. ${ }^{37}$ In recent theoretical investigations the anisotropies in the effective $g$ factor, ${ }^{38}$ spin polarizations, ${ }^{39}$ and controlled spin rotations induced by Rashba-type spin-orbit coupling in a spin-FET setup $^{23}$ have been considered. In the present work we investigate the spin-Hall effect for heavy holes in III-V zinc blende semiconductor quantum wells.

This paper is organized as follows. In Sec. II we discuss the Rashba model for heavy holes in III-V semiconductor quantum wells. As an aside, we also give explicit expressions for the Fermi momenta and the densities of holes in the different dispersion branches as a function of the spin-orbit coupling parameter and the total hole density. Apart from the present investigations, we expect these results to be also of 
relevance for the interpretation of possible Shubnikov-de Haas measurements ${ }^{40,41}$ in such hole systems. Section III is devoted to the discussion of our results on spin-Hall transport, and we close with conclusions in Sec. IV.

\section{RASHBA SPIN-ORBIT COUPLING FOR HEAVY HOLES}

We consider the following single-particle Hamiltonian incorporating spin-orbit coupling due to structure-inversion asymmetry for heavy holes in III-V semiconductor quantum wells of appropriate growth geometry ${ }^{20-24}$

$$
\mathcal{H}=\frac{\vec{p}^{2}}{2 m}+i \frac{\alpha}{2 \hbar^{3}}\left(p_{-}^{3} \sigma_{+}-p_{+}^{3} \sigma_{-}\right),
$$

using the notations $p_{ \pm}=p_{x} \pm i p_{y}, \sigma_{ \pm}=\sigma_{x} \pm i \sigma_{y}$, where $\vec{p}, \vec{\sigma}$ denote the hole momentum operator and Pauli matrices, respectively. These Pauli matrices operate on the total angular momentum states with spin projection $\pm 3 / 2$ along the growth direction; in this sense they represent a pseudospin degree of freedom rather than a genuine spin $1 / 2$. In the above equation, $m$ is the heavy-hole mass, and $\alpha$ is Rashba spin-orbit coupling coefficient due to structure inversion asymmetry across the quantum well grown along the [001] direction chosen as the $z$ axis. For a symmtrically grown quantum well, the coefficient $\alpha$ is essentially proportional to an electric field applied across the well and therefore experimentally tunable. $^{20-22}$ This Hamiltonian has two dispersion branches given by

$$
\varepsilon_{ \pm}(k)=\frac{\hbar^{2} k^{2}}{2 m} \pm \alpha k^{3}
$$

with eigenfunctions

$$
\langle\vec{r} \mid \vec{k}, \pm\rangle=\frac{e^{i \vec{k} \cdot \vec{r}}}{\sqrt{A}} \frac{1}{\sqrt{2}}\left(\begin{array}{c}
1 \\
\mp i\left(k_{x}+i k_{y}\right)^{3} / k^{3}
\end{array}\right),
$$

where $\vec{k}$ is the hole lattice momentum and $A$ is the area of the system. We note that the validity of above model given by the Hamiltonian (1) is restricted to sufficiently small wave numbers and densities. In fact, the lower of the two dispersion branches (2) is (for $\alpha>0$ ) not bounded from below for large wave numbers and has non-negative eigenenergies only for $k \leqslant \hbar^{2} / 2 m \alpha$ with a maximum at $k=\hbar^{2} / 3 m \alpha$. The unbounded decrease of the single-particle energies with increasing wave number for $k>\hbar^{2} / 3 m \alpha$ is clearly an unphysical feature of the model. Therefore, the following considerations are restricted to sufficiently small densities such that (at zero temperature) only states with wave numbers $k \leqslant \hbar^{2} / 3 m \alpha$ are occupied.

For a given Fermi energy $\varepsilon_{f}$ and vanishing temperature, the above two dispersion branches give rise to two different Fermi wave numbers $k_{f}^{ \pm}$fulfilling

$$
\varepsilon_{f}=\frac{\hbar^{2}\left(k_{f}^{ \pm}\right)^{2}}{2 m} \pm \alpha\left(k_{f}^{ \pm}\right)^{3}
$$

with $k_{f}^{+}<k_{f}^{-}\left(k_{f}^{+}>k_{f}^{-}\right)$for positive (negative) $\alpha$. Subtracting these two equations one finds

$$
0=\frac{\hbar^{2}}{2 m}\left[\left(k_{f}^{+}\right)^{2}-\left(k_{f}^{-}\right)^{2}\right]+\alpha\left[\left(k_{f}^{+}\right)^{3}+\left(k_{f}^{-}\right)^{3}\right]
$$

or, cancelling a factor of $\left(k_{f}^{+}+k_{f}^{-}\right)$,

$$
k_{f}^{+} k_{f}^{-}=-\frac{\hbar^{2}}{2 m \alpha}\left(k_{f}^{+}-k_{f}^{-}\right)-\left(k_{f}^{+}-k_{f}^{-}\right)^{2} .
$$

Note that in the relations (5) and (6) the Fermi energy does not enter explicitly. In fact, the quantity which can be immediately controlled experimentally is not the Fermi energy but the hole density $n$ given by

$$
n=\frac{1}{4 \pi}\left[\left(k_{f}^{+}\right)^{2}+\left(k_{f}^{-}\right)^{2}\right] .
$$

Combining Eqs. (6) and (7), one obtains

$$
\begin{gathered}
k_{f}^{+}-k_{f}^{-}=-\frac{\hbar^{2}}{2 m \alpha}\left[1-\sqrt{1-\left(\frac{2 m \alpha}{\hbar^{2}}\right)^{2} 4 \pi n}\right], \\
k_{f}^{+} k_{f}^{-}=4 \pi n-\left(\frac{\hbar^{2}}{2 m \alpha}\right)^{2}\left[1-\sqrt{1-\left(\frac{2 m \alpha}{\hbar^{2}}\right)^{2} 4 \pi n}\right] .
\end{gathered}
$$

Note that for $k_{f}^{ \pm} \leqslant \hbar^{2} / 3 m \alpha$ the radicand in the above equations is always positive. Using

$$
4 \pi n=\left(k_{f}^{+}+k_{f}^{-}\right)^{2}-2 k_{f}^{+} k_{f}^{-}
$$

one derives from the above equations the following explicit expression for $k_{f}^{ \pm}$as a function of the density $n$ and the Rashba parameter $\alpha$ :

$$
\begin{aligned}
k_{f}^{ \pm}= & \mp \frac{1}{2} \frac{\hbar^{2}}{2 m \alpha}\left[1-\sqrt{1-\left(\frac{2 m \alpha}{\hbar^{2}}\right)^{2} 4 \pi n}\right] \\
& +\left\{-\frac{1}{2}\left(\frac{\hbar^{2}}{2 m \alpha}\right)^{2}\left[1-\sqrt{1-\left(\frac{2 m \alpha}{\hbar^{2}}\right)^{2} 4 \pi n}\right]+3 \pi n\right\}^{1 / 2} .
\end{aligned}
$$

The difference $\Delta n$ of densities of holes in the two dispersion branches

$$
\Delta n=\frac{1}{4 \pi}\left[\left(k_{f}^{+}\right)^{2}-\left(k_{f}^{-}\right)^{2}\right],
$$

can be expressed as

$$
\begin{aligned}
\Delta n= & \frac{-1}{4 \pi} \frac{\hbar^{2}}{2 m \alpha}\left[1-\sqrt{1-\left(\frac{2 m \alpha}{\hbar^{2}}\right)^{2} 4 \pi n}\right] \\
& \times\left\{-2\left(\frac{\hbar^{2}}{2 m \alpha}\right)^{2}\left[1-\sqrt{1-\left(\frac{2 m \alpha}{\hbar^{2}}\right)^{2} 4 \pi n}\right]+12 \pi n\right\}^{1 / 2} .
\end{aligned}
$$

Such a difference in densities in the two dispersion branches could be experimentally detected in Shubnikov-de Haas measurements, as it was shown earlier for the case of electrons. ${ }^{40,41}$ We note that the above results for the Fermi momenta and $\Delta n$ depend only on the total hole densty $n$ and length scale $m \alpha / \hbar^{2}$ given by the Rashba coupling. ${ }^{42}$ Winkler et $a .^{22}$ have studied both theoretically and experimentally 


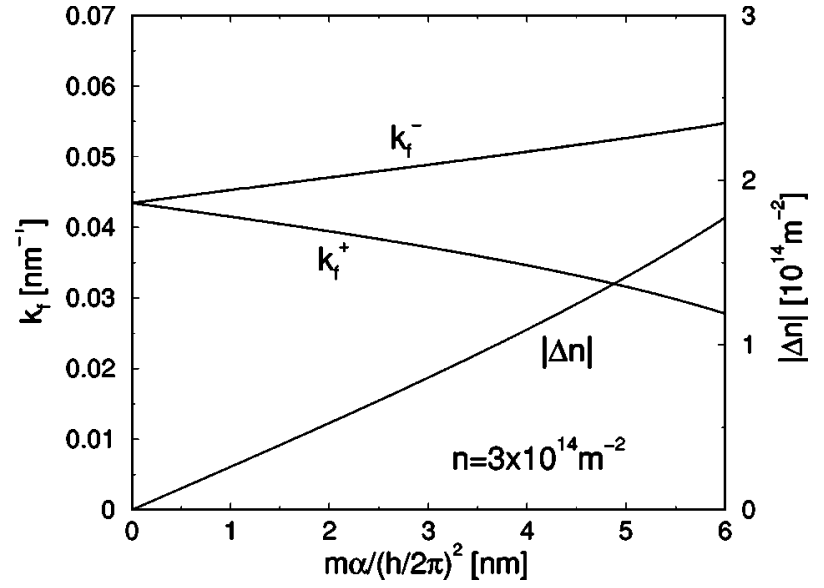

FIG. 1. The Fermi wave numbers $k_{f}^{ \pm}$and the difference $\Delta n$ of hole densities in the two dispersion branches as a function of the characteristic length $m \alpha / \hbar^{2}$ of the Rashba coupling at a total hole density of $n=3 \times 10^{14} \mathrm{~m}^{-2}$.

the magnitude of the Rashba spin orbit coupling in GaAsbased quantum well samples with heavy-hole densities of a few $10^{14} \mathrm{~m}^{-2}$ and have found typical values for the characteristic length scale $m \alpha / \hbar^{2}$ of a few nanometers. In Fig. 1 we have plotted the Fermi wave numbers $k_{f}^{ \pm}$and the difference $\Delta n$ of holes in the two dispersion branches as a function of $m \alpha / \hbar^{2}$ at a total hole density of $n=3 \times 10^{14} \mathrm{~m}^{-2}$.

On the other hand, solving Eq. (8) for $\alpha$ gives a convenient expression for the Rashba coefficient as a function of the total hole density and the difference of the Fermi wave numbers

$$
\alpha=\frac{\hbar^{2}}{2 m} \frac{-2\left(k_{f}^{+}-k_{f}^{-}\right)}{\left(k_{f}^{+}-k_{f}^{-}\right)^{2}+4 \pi n} .
$$

Finally, writing Eq. (4) in the form

$$
\varepsilon_{f}\left(\frac{1}{k_{f}^{ \pm}}\right)^{3}=\frac{\hbar^{2}}{2 m} \frac{1}{k_{f}^{ \pm}} \pm \alpha
$$

one obtains by adding these two equations

$$
\frac{2 m}{\hbar^{2}} \varepsilon_{f}\left[\left(\frac{1}{k_{f}^{+}}-\frac{1}{k_{f}^{-}}\right)^{2}+\frac{1}{k_{f}^{+} k_{f}^{-}}\right]=1,
$$

which does not explicitly depend on the Rashba parameter $\alpha$. From this relation, it follows with the help of Eq. (6)

$$
\varepsilon_{f}=\alpha \frac{\left(k_{f}^{+} k_{f}^{-}\right)^{2}}{k_{f}^{-}-k_{f}^{+}} .
$$

Using Eqs. (8) and (9) it is straightforward to obtain from this expression an explicit relation between the Fermi energy $\epsilon_{f}$ and the hole density $n$ at a given Rashba parameter $\alpha$.

\section{SPIN-HALL TRANSPORT}

We now investigate the possibilty of spin-Hall transport of heavy holes in III-V semiconductor quantum wells in the presence of Rashba-type spin-orbit coupling. We are inter- ested in spin currents (as opposed to charge currents) as the linear response of the system to an electric field applied in the plane of the well. As we shall see below, in such a system the spin current is always perpendicular to the driving electric field and therefore Hall type. We concentrate on the case of zero temperature.

The single-particle spin current operator is defined by

$$
\vec{j} S, z=\frac{3 \hbar}{2} \frac{1}{2}\left(\sigma^{z} \vec{v}+\vec{v} \sigma^{z}\right)=\frac{3 \hbar}{2} \frac{\vec{p}}{m} \sigma^{z},
$$

where the factor of $3 / 2$ reflects the angular momentum quantum numbers of the heavy holes. The hole velocity operator reads $\vec{v}=i[\mathcal{H}, \vec{r}] / \hbar$ with $\vec{r}$ being the position operator or, in terms of components,

$$
\begin{aligned}
& v_{x}=\frac{p_{x}}{m}+\frac{\alpha}{\hbar^{3}}\left[6 p_{x} p_{y} \sigma^{x}+3\left(p_{y}^{2}-p_{x}^{2}\right) \sigma^{y}\right], \\
& v_{y}=\frac{p_{y}}{m}+\frac{\alpha}{\hbar^{3}}\left[3\left(p_{x}^{2}-p_{y}^{2}\right) \sigma^{x}+6 p_{x} p_{y} \sigma^{y}\right] .
\end{aligned}
$$

A rigorous expression for the spin conductivity, i.e., the linear transport coefficient for spin currents driven by a spatially homogeneous in-plane electric field, is given by the Kubo formula with full frequency dependence for an electric field ${ }^{43}$

$$
\begin{aligned}
\sigma_{x y}^{S, z}(\omega)= & \frac{e}{A(\omega+i \eta)} \int_{0}^{\infty} e^{i(\omega+i \eta) t} \sum_{\vec{k}, \mu} f\left[\varepsilon_{\mu}(\vec{k})\right] \\
& \times\left\langle\vec{k}, \mu\left|\left[j_{x}^{S, z}(t), v_{y}(0)\right]\right| \vec{k}, \mu\right\rangle,
\end{aligned}
$$

where we have concentrated on the off-diagonal $x y$ components. Moreover, we have assumed zero temperature $T=0$ and noninteracting carriers, which allows us to formulate the two-body Green's function entering the conductivity Kubo formula in terms of single-particle operators. $e=|e|$ is the elementary charge and $f\left[\varepsilon_{\mu}(\vec{k})\right]$ is the $T=0$ Fermi distribution function for energy $\varepsilon_{\mu}(\vec{k})$ at wave vector $\vec{k}$ in the dispersion branch $\mu \in\{+,-\}$. The spin-current operator (in the Dirac picture) for spin moment polarized along the $z$ direction and flowing in the $x$ direction is given by

$$
j_{x}^{S, z}(t)=e^{i \mathcal{H} t / \hbar} j_{x}^{S, z}(0) e^{-i \mathcal{H} t / \hbar}=\frac{3 \hbar}{2 m} \sigma^{z}(t) p_{x}(t)
$$

From now on we will assume the Hamiltonian generating the above time evolution to include also scattering potentials from static random impurities being present in the quantum well. The right-hand side of Eq. (21) is to be understood in the limit of vanishing imaginary part $\eta>0$ in the frequency argument. This imaginary part in the frequency reflects the fact that the external electric field is assumed to be switched on adiabatically starting from the infinite past of the system, and it also ensures causality properties of the retarded Green's function occurring in Eq. (21). In general the limiting process $\eta \rightarrow 0$ does not commute with other limits and, in particular, the dc limit $\omega \rightarrow 0$ has to be taken with care. ${ }^{43}$ In the presence of random impurity scattering, the retarded twobody Green's function in Eq. (21) will generically have a 
frequency argument with positive imaginary part. ${ }^{43}$ In this case the limit $\eta \rightarrow 0$ is unproblematic, and the imaginary part of the frequency argument is just due to impurity scattering and/or other (many-body) effects. For the present problem of impurity scattering of noninteracting carriers being subject to Rashba-type spin-orbit coupling, the resulting imaginary part $\eta>0$ in the frequency argument is given, to lowest order in the Rashba coefficient and the impurity potential, by the inverse of the momentum relaxation time. This is certainly a very intuitive result; the formal arguments leading to it is completely analogous to the ones used in Ref. 11 and can be given along the following lines: In lowest order in the spinorbit coupling and the impurity scattering the time-dependent spin-current operator reads

$$
j_{x}^{S, z}(t) \approx \frac{3 \hbar}{2 m} \sigma_{0}^{z}(t) p_{x}^{0}(t),
$$

where the time evolution of $\sigma_{0}^{z}$ is only due to the Hamiltonian (1), while $p_{x}^{0}(t)$ contains the impurity scattering but not the spin-orbit coupling. Now it is useful to note that, in order to compute the expectation values in the Kubo formula Eq. (21), only matrix elements of the time-dependent momentum operator $p_{x}^{0}(t)$ which are diagonal in the wave vector index are needed. This enables to apply superoperator techniques developed in Refs. 44 yielding

$$
\left[p_{x}^{0}(t)\right]_{\vec{k} \cdot \vec{k}} \approx\left[e^{-\Omega_{0} t} p_{x}^{0}(0)\right]_{\vec{k} \cdot \vec{k}}=\left[e^{-t / \tau} p_{x}^{0}(0)\right]_{\vec{k} \cdot \vec{k}},
$$

where $\Omega_{0}$ is the scattering master operator in lowest order of the scattering potential. ${ }^{44}$ This operator is the same as it occurs as the scattering term in the usual Boltzmann equation when evaluated in lowest oder via Fermi's golden rule. Thus, Eq. (24) describes the usual momentum relaxation due to static impurities in lowest order in the scattering potential. For impurity potentials being isotropic in real space, the momentum $p_{x}$ is an exact eigenfunction of $\Omega_{0}$, and the eigenvalue is given by the well-known inverse momentum relaxation time $1 / \tau(\varepsilon)$ (Refs. 44 and 45) which in general depends on the energy $\varepsilon(\vec{k})$. To lowest order in the Rashba coupling, this energy argument can be replaced with the Fermi energy in the absence of spin-orbit interaction. We note that this momentum relaxation rate $1 / \tau$ is the same as obtained in the standard diagrammatic approach and thus contains the vertex correction for particle transport. ${ }^{43}$ However, this vertex correction vanishes for short-range isotropic scatterers.

The question of vertex corrections to the spin-Hall transport was also examined very recently by Inoue, Bauer, and Molenkamp, ${ }^{16}$ and by Dimitrova ${ }^{19}$ for the case of electrons being subject to Rashba spin-orbit interaction. There the authors reach the conclusion that for small but finite disorder the spin-Hall conductivity should identically vanish due to vertex corrections, while it has its "universal value" of $e / 8 \pi$ in the case of a perfectly clean system. ${ }^{10-12}$ Moreover, Murakami ${ }^{9}$ has studied vertex correction to spin-Hall transport of holes in bulk $p$-type semiconductors described by a Luttinger Hamiltonian. ${ }^{32}$ There the author concludes that vertex correction vanish identically, validating, the results of Ref. 31, and ascribes this observation to the fact that the underlying Hamiltonian is invariant under inversion of mo- menta, in contrast to the Rashba Hamiltonian. These technically rather involved issues are to the opinion of the present authors not entirely settled yet and presently under investigation.

Let us now turn to the evaluation of the spin-Hall conductivity using the aforementioned approximations. A straightforward calculation yields

$$
\sigma_{x y}^{S, z}(\omega)=-\sigma_{y x}^{S, z}(\omega)=-\frac{e}{\pi} \frac{9}{4} \frac{\alpha}{m} \int_{k_{f}^{+}}^{k_{f}^{-}} d k \frac{k^{4}}{\left(\omega+\frac{i}{\tau}\right)^{2}-\left(\frac{2 \alpha k^{3}}{\hbar}\right)^{2}},
$$

where, according to the above arguments, the imaginary part of the frequency argument is given by the momentum relaxation rate $1 / \tau$. Moreover, the longitudinal spin conductivities $\sigma_{x x}^{S, z}, \sigma_{y y}^{S, z}$ turn out ot be identically zero. This is similar to the case of electrons in a quantum well being subject to spinorbit coupling of either the Rashba or the Dresselhaus type. ${ }^{10-12}$ There a longitudinal spin conductivity occurs only if both the Rashba and the Dresselhaus coupling are present. $^{12}$

The remaning integral in the above expression (25) is elementary; however, it leads to a rather cumbersome expression which shall not be given here. In the dc limit $\omega=0$, the energy scale of the impurity scattering $\hbar / \tau$ has to be compared with the "Rashba energy" $\varepsilon_{R}=\alpha\left(k_{f}^{0}\right)^{3}$, where $k_{f}^{0}$ $=\sqrt{2 m \varepsilon_{f} / \hbar^{2}}$ is the Fermi wave number for vanishing spinorbit coupling, which is a typical value for $k$ in the inegration in Eq. (25). If the impurity scattering dominates over the Rashba coupling $\hbar / \tau \gg \varepsilon_{R}$, the spin-Hall conductivity vanishes with the leading order correction given by

$$
\sigma_{x y}^{S, z}(0)=\frac{e}{\pi} \frac{9}{20} \frac{\alpha}{m} \tau^{2}\left[\left(k_{f}^{-}\right)^{5}-\left(k_{f}^{+}\right)^{5}\right]+O\left[\left(\frac{\varepsilon_{R}}{\hbar / \tau}\right)^{4}\right],
$$

where the Fermi wave numbers are given by Eq. (11). In the opposite case $\varepsilon_{R} \gg \hbar / \tau$, the leading contribution to the spinHall conductivity reads

$$
\sigma_{x y}^{S, z}(0)=\frac{e}{\pi} \frac{9}{16} \frac{\hbar^{2}}{m \alpha}\left(\frac{1}{k_{f}^{+}}-\frac{1}{k_{f}^{-}}\right)+O\left[\left(\frac{\hbar / \tau}{\varepsilon_{R}}\right)^{4}\right] .
$$

Note that this result for the spin-Hall conductivity depends only on the length scale $m \alpha / \hbar^{2}$ of the Rashba coupling and the total hole density $n$, but not separately on quantities such as the Fermi energy and the effective heavy hole mass. If $m \alpha / \hbar^{2}$ is small against the inverse square root of the total hole density (but still fulfilling $\varepsilon_{R} \gg \hbar / \tau$ ), the spin-Hall conductivity approaches a value of $\sigma_{x y}^{S, z}=9 e / 8 \pi$. This is the case if $\hbar / \tau \ll \varepsilon_{R} \ll \varepsilon_{f}$. This above value should be compared with the universal value of $e / 8 \pi$ found for the spin-Hall conductivity of electrons being subject to a Rashba coupling dominating possible impurity scattering. ${ }^{10,11}$ Thus, in this limit, the hole spin-Hall conductivity is enhanced compared to the case of electrons by a factor of 9 , which is partially due to the larger angular momentum of the heavy holes. In Fig. 2 we have plotted the spin-Hall conductivity for dominating Rashba coupling $\left[\varepsilon_{R} \gg \hbar / \tau\right.$, see Eq. (27)] as a function of $m \alpha / \hbar^{2}$ at a total hole density of $n=3 \times 10^{14} \mathrm{~m}^{-2}$. As seen 


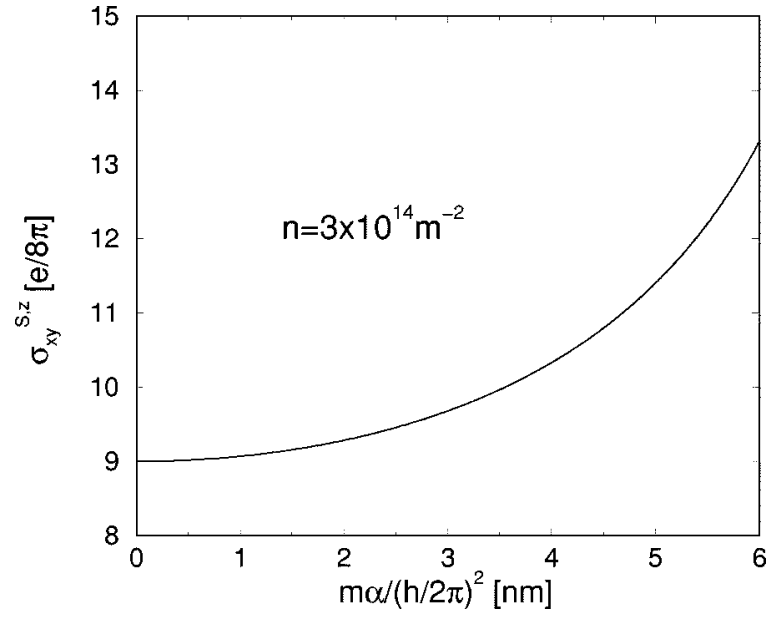

FIG. 2. The spin-Hall condcutivity for dominating Rashba coupling $\left[\varepsilon_{R} \gg \hbar / \tau\right.$, see Eq. (27)] as a function of $m \alpha / \hbar^{2}$ at a total hole density of $n=3 \times 10^{14} \mathrm{~m}^{-2}$.

there, the spin-Hall conductivity starts out at $\sigma_{x y}^{S, z}=9(e / 8 \pi)$ and increases with increasing Rashba coupling.

\section{CONCLUSIONS}

We have studied spin transport of heavy holes in III-V semiconductor quantum wells in the presence of spin-orbit coupling of the Rashba type due to structure-inversion asymmetry. Similarly to the case of electrons, the longitudinal spin conductivity vanishes, whereas the off-diagonal elements of the spin-conductivity tensor are finite giving rise to an intrinsic spin-Hall effect. For a clean system we find a closed expression for the spin-Hall conductivity depending on the length scale of the Rashba coupling and the hole density. In this limit the spin-Hall conductivity is enhanced compared to its value for electron systems. For dirtier $p$-doped quantum wells when the impurity scattering dominates the spin-orbit coupling, the spin-Hall conductivity naturally vanishes as also found previously for the case of electrons. ${ }^{11,12}$ As an aside, we give explicit expressions for the Fermi momenta and the densities of holes in the different dispersion branches as a function of the spin-orbit coupling parameter and the total hole density. These results are expected to be helpful for the interpretation of possible Shubnikov-de Haas experiments aiming at the detection of the Rashba spin splitting.

Note added. After submission of this paper a preprint by Wunderlich et $a l .{ }^{46}$ appeared reporting on an experimental observation of the spin Hall effect in $p$-doped GaAs quantum wells as studied here.

\section{ACKNOWLEDGMENTS}

We thank J. Carlos Egues, S. Erlingsson, C.-M. Hu, D. Saraga, O. Shalaev, and R. Winkler for stimulating discussions. This work was supported by the NCCR Nanoscience, the Swiss NSF, DARPA, ARO, ONR, and the EU Spintronics RTN.
${ }^{1}$ S. A. Wolf, D. D. Awschalom, R. A. Buhrman, J. M. Daughton, S. von Molnar, M. L. Roukes, A. Y. Chtchelkanova, and D. M. Treger, Science 294, 1488 (2001).

${ }^{2}$ Semiconductor Spintronics and Quantum Computation, edited by D. D. Awschalom, D. Loss, and N. Samarth (Springer, Berlin, 2002).

${ }^{3}$ E. I. Rashba, Physica E (Amsterdam) 20, 189 (2004).

${ }^{4}$ S. Murakami, N. Nagaosa, and S. C. Zhang, Science 301, 1348 (2003); Phys. Rev. B 69, 235206 (2004).

${ }^{5}$ D. Culcer, J. Sinova, N. A. Sinitsyn, T. Jungwirth, A. H. MacDonald, and Q. Niu, Phys. Rev. Lett. 93, 046602 (2004).

${ }^{6} \mathrm{~J} . \mathrm{Hu}, \mathrm{B}$. A. Bernevig, and C. Wu, cond-mat/0310093 (unpublished)

${ }^{7}$ B. A. Bernevig, J. P. Hu, E. Mukamel, and S. C. Zhang, Phys. Rev. B 70, 113301 (2004).

${ }^{8} \mathrm{~L}$. Hu, J. Gao, and S.-Q. Shen, cond-mat/0401231.

${ }^{9}$ S. Murakami, Phys. Rev. B 69, 241202(R) (2004).

${ }^{10}$ J. Sinova, D. Culcer, Q. Niu, N. A. Sinitsyn, T. Jungwirth, and A. H. MacDonald, Phys. Rev. Lett. 92, 126603 (2004).

${ }^{11}$ J. Schliemann and D. Loss, Phys. Rev. B 69, 165315 (2004).

${ }^{12}$ N. A. Sinitsyn, E. M. Hankiewicz, W. Teizer, and J. Sinova, Phys. Rev. B 70, 081312(R) (2004).

${ }^{13}$ S.-Q. Shen, Phys. Rev. B 70, 081311 (2004).

${ }^{14}$ A. A. Burkov, A. S. Nunez, and A. H. MacDonald, Phys. Rev. B 70, 155308 (2004).

${ }^{15}$ E. I. Rashba, Phys. Rev. B 68, 241315(R) (2003).
${ }^{16}$ J. Inoue, G. E. W. Bauer, and L. W. Molenkamp, Phys. Rev. B 70, 041303(R) (2004).

${ }^{17}$ S.-Q. Shen, M. Ma, X. C. Xie, and F. C. Zhang, Phys. Rev. Lett. 92, 256603 (2004).

${ }^{18}$ E. I. Rashba, Phys. Rev. B 70, 161201 (2004).

${ }^{19}$ O. V. Dimitrova, cond-mat/0405339 (unpublished).

${ }^{20}$ L. G. Gerchikov and A. V. Subashiev, Sov. Phys. Semicond. 26, 73 (1992).

${ }^{21}$ R. Winkler, Phys. Rev. B 62, 4245 (2000).

${ }^{22}$ R. Winkler, H. Noh, E. Tutuc, and M. Shayegan, Phys. Rev. B 65, 155303 (2002).

${ }^{23}$ M. G. Pala, M. Governale, J. König, and U. Zülicke, Europhys. Lett. 65, 850 (2004); M. G. Pala, M. Governale, J. König, U. Zülicke, and G. Iannaccone, Phys. Rev. B 69, 045304 (2004).

${ }^{24}$ For spin-orbit effects on holes in quantum wells due to bulkinversion asymmetry (as opposed to structure-inversion asymmetry studied here) see E. I. Rashba and E. Y. Sherman, Phys. Lett. A 129, 175 (1988).

${ }^{25}$ M. I. Dyakonov and V. I. Perel, Phys. Lett. 35A, 459 (1971).

${ }^{26}$ J. E. Hirsch, Phys. Rev. Lett. 83, 1834 (1999).

${ }^{27}$ F. Meier and D. Loss, Phys. Rev. Lett. 90, 167204 (2003).

${ }^{28}$ E. I. Rashba, Fiz. Tverd. Tela (Leningrad) 2, 1224 (1960) [Sov. Phys. Solid State 2, 1109 (1960)]; Y. A. Bychkov and E. I. Rashba, J. Phys. C 17, 6039 (1984).

${ }^{29}$ G. Dresselhaus, Phys. Rev. 100, 580 (1955).

${ }^{30}$ J. Schliemann, J. C. Egues, and D. Loss, Phys. Rev. Lett. 90, 
146801 (2003).

${ }^{31}$ J. Schliemann and D. Loss, Phys. Rev. B 68, 165311 (2003).

${ }^{32}$ J. M. Luttinger, Phys. Rev. 102, 1030 (1956).

${ }^{33}$ R. Winkler, Phys. Rev. B 70, 125301 (2004).

${ }^{34}$ E. Tutuc, E. P. De Poortere, S. J. Papadakis, and M. Shayegan, Phys. Rev. Lett. 86, 2858 (2001).

${ }^{35}$ S. J. Papadakis, E. P. De Poortere, H. C. Manoharan, M. Shayegan, and R. Winkler, Science 283, 2056 (1999); S. J. Papadakis, E. P. De Poortete, M. Shayegan, and R. Winkler, Physica E (Amsterdam) 9, 31 (2001).

${ }^{36}$ S. J. Papadakis, E. P. De Poortere, H. C. Manoharan, J. B. Yau, M. Shayegan, and S. A. Lyon, Phys. Rev. B 65, 245312 (2002).

${ }^{37}$ S. J. Papadakis, E. P. De Poortere, M. Shayegan, and R. Winkler, Phys. Rev. Lett. 84, 5592 (2000).

${ }^{38}$ R. Winkler, S. J. Papadakis, E. P. De Poortere, and M. Shayegan, Phys. Rev. Lett. 85, 4574 (2000).

${ }^{39}$ R. Winkler, cond-mat/0401067 (unpublished).

${ }^{40}$ J. Luo, H. Munekata, F. F. Fang, and P. J. Stiles, Phys. Rev. B 38, 10142 (1988).

${ }^{41}$ G. Engels, J. Lange, T. Schäpers, and H. Lüth, Phys. Rev. B 55, R1958 (1997); T. Schäpers, G. Engels, J. Lange, T. Klocke, H.
Hollfelder, and H. Lüth, J. Appl. Phys. 83, 4234 (1998).

${ }^{42}$ We note that for Rashba coupling of conduction band electrons the characteristic length scale is given by $\hbar^{2} / m \tilde{\alpha}$ where $\widetilde{\alpha}$ is the electron Rashba parameter. This quantity is naively speaking just the "inverse" of the characteristic length $m \alpha / \hbar^{2}$ for holes. This difference is due to the different functional form of the Rashba spin-orbit contribution for electrons and holes (linear versus cubic dependence on the momenta). Moreover, the length scale $m \alpha / \hbar^{2}$ introduced here is different from the spin precession length used in Ref. 23 which involves the Fermi energy.

${ }^{43}$ G. Mahan, Many-Particle Physics, 3rd ed. (Kluwer, New York, 2000).

${ }^{44}$ D. Loss, Physica A 139, 505 (1986); see also 139, 526 (1986); D. Loss and A. Thellung, ibid. 144, 17 (1987).

${ }^{45}$ See, e.g., H. Smith and H. H. Jensen, Transport Phenomena (Clarendon Press, Oxford, 1989); J. M. Ziman, Principles of the Theory of Solids (Cambridge University Press, Cambridge, 1972).

${ }^{46}$ J. Wunderlich, B. Kästner, J. Sinova, and T. Jungwirth, cond-mat/ 0410295 (unpublished). 